\title{
Some Aspects on Filter Design for Target Tracking
}

\author{
Bertil Ekstrand \\ Ekelundsvägen 22, 42942 Särö, Sweden \\ Correspondence should be addressed to Bertil Ekstrand, bertilekstrand@telia.com
}

Received 6 October 2011; Revised 30 January 2012; Accepted 30 January 2012

Academic Editor: Wen Yu

Copyright ( 12012 Bertil Ekstrand. This is an open access article distributed under the Creative Commons Attribution License, which permits unrestricted use, distribution, and reproduction in any medium, provided the original work is properly cited.

Tracking filter design is discussed. It is argued that the basis of the present stochastic paradigm is questionable. White process noise is not adequate as a model for target manoeuvring, stochastic least-square optimality is not relevant or required in practice, the fact that requirements are necessary for design is ignored, and root mean square (RMS) errors are insufficient as performance measure. It is argued that there is no process noise and that the covariance of the assumed process noise contains the design parameters. Focus is on the basic tracking filter, the Kalman filter, which is convenient for clarity and simplicity, but the arguments and conclusions are relevant in general. For design the possibility of an observer transfer function approach is pointed out. The issues can also be considered as a consequence of the fact that there is a difference between estimation and design. The $\alpha-\beta$ filter is used for illustration.

\section{Introduction}

To obtain kinematic components of a moving target such as position, velocity, and acceleration, the predominant paradigm in target tracking is to use a stochastic estimation approach. A moving target is described by a state-space dynamic system driven by white process noise, and the state is estimated in mean-square sense using noisy position measurements. As we know, models of this kind are the basis of Kalman filters, and, indeed, the Kalman filter is the basic algorithm in target tracking, either as a single filter or as a part of other algorithms such as in different adaptive settings. Estimation algorithms within this stochastic framework have also been developed to deal with nonlinearities, multiple target tracking, and measurement association.

Much research has been devoted to different variations of this approach, see [1-5] and further references therein. However, when applied to practical applications some questions arise. For example, it is not realistic to consider white process noise as a model for target manoeuvring, and a mean-square criterion is usually not adequate in practice. Furthermore, requirements are necessary for design, but this important fact is ignored in tracking. The tracking literature is noteworthy in paying no attention to these issues. This somewhat surprising situation is the reason for the present paper.
The issues are discussed with the Kalman filter in mind, and the well-known $\alpha-\beta(-\gamma)$ archetype tracking filters are used as examples. This is convenient for simplicity and clarity and is also of basic interest considering the importance of the Kalman filter in tracking. However, the arguments can be applied to more complex algorithms than a single Kalman filter. In fact, in the stochastic paradigm, different tracking filters are obtained by using the same approach: estimation algorithms are proposed based on stochastic models with white process noise, and requirements are ignored. Discussions of the Kalman filter in this respect will then be relevant for other tracking algorithms.

In any case, for the particular issues discussed, it will be clear that most of the arguments are valid or have correspondences in general, although this is not pointed out in all places.

In Section 2 basic Kalman filter notations are given. As a suitable background, models underlying the $\alpha-\beta(-\gamma)$ filters are presented in Section 3. Requirements are discussed in Section 4 and Kalman filter properties related to target tracking in Section 5. Filter evaluations are discussed in Section 6 and the IMM adaptive approach in Section 7. Some further aspects are given in Section 8. The $\alpha-\beta$ filter is used for illustration in Section 9. Finally, comparison with optimal control is done in the Appendix. 


\section{The Kalman Filter}

For easy reference basic Kalman filter notations are given. Consider the standard linear discrete-time system

$$
\begin{gathered}
x(k+1)=F x(k)+v(k), \\
y(k)=H x(k)+w(k) .
\end{gathered}
$$

As usual $x(k)$ is the state, $y(k)$ the measurement, and $k=0,1,2, \ldots$ a time index, that is, the sampling time instants $t_{k}=k T$ where $T$ is the sampling interval. $v(k)$ and $w(k)$ are zero-mean white noise sequences, the process and measurement noise respectively, with covariance

$$
E\left[v(k) v(k)^{\prime}\right]=Q ; \quad E\left[w(k) w(k)^{\prime}\right]=R .
$$

The Kalman filter for this system is the algorithm

$$
\begin{aligned}
\hat{x}(k+1 \mid k+1)= & \hat{x}(k+1 \mid k) \\
& +L(k+1)[y(k+1)-H \hat{x}(k+1 \mid k)], \\
\hat{x}(k+1 \mid k)= & F \hat{x}(k \mid k),
\end{aligned}
$$

where $\hat{x}(k \mid k)$ is the updated state estimate and $\hat{x}(k+1 \mid k)$ is the one-step-ahead prediction of the state. $F, H, Q, R$ are assumed known; they enter into the Riccati equation, the solution of which determines the prediction error covariance and by that the gain $L(k)$. For a time-invariant system, the solution of the Riccati equation converges to a unique steadystate covariance matrix under the usual observability and controllability conditions, which gives a constant gain. The algorithm (3) is called a Kalman filter only if $L(k)$ is obtained from the Riccati equation. If a gain other than the Kalman gain is used, we may think of (3) as an observer.

\section{Target Motion Models}

Models underlying tracking filters are often of second or third order with state vector $x(k)=\left[x_{1}(k) x_{2}(k)\right]^{\prime}$ and $x(k)=\left[\begin{array}{lll}x_{1}(k) & x_{2}(k) & x_{3}(k)\end{array}\right]^{\prime}$, respectively, where $x_{1}$ is position, $x_{2}$ velocity, and $x_{3}$ acceleration. $x_{1}$ is typically a Cartesian or an angle position and is usually the measured variable. For Kalman filters a common model of second order is

$$
\begin{gathered}
F=\left[\begin{array}{ll}
1 & T \\
0 & 1
\end{array}\right], \quad H=\left[\begin{array}{ll}
1 & 0
\end{array}\right], \\
Q=\left[\begin{array}{cc}
\frac{T^{2}}{4} & \frac{T}{2} \\
\frac{T}{2} & 1
\end{array}\right] \sigma_{v}^{2} T^{2}, \quad R=\sigma_{w}^{2},
\end{gathered}
$$

where $\sigma_{w}$ is the standard deviation of the white sequence $w(k) . v(k)$ is the target acceleration, assumed to be constant during a sampling interval where the interval values are a white sequence with standard deviation $\sigma_{v}$. For the thirdorder case a corresponding model is

$$
\begin{aligned}
F & =\left[\begin{array}{ccc}
1 & T & \frac{T^{2}}{2} \\
0 & 1 & T \\
0 & 0 & 1
\end{array}\right], \quad H=\left[\begin{array}{lll}
1 & 0 & 0
\end{array}\right] . \\
Q & =\left[\begin{array}{ccc}
\frac{T^{4}}{4} & \frac{T^{3}}{2} & \frac{T^{2}}{2} \\
\frac{T^{3}}{2} & T^{2} & T \\
\frac{T^{2}}{2} & T & 1
\end{array}\right] \sigma_{v}^{2}, \quad R=\sigma_{w}^{2} .
\end{aligned}
$$

In this model, it is the target acceleration increment during a sampling interval that is random, given by the white sequence $v(k)$. The steady-state Kalman filters for these models are the so called $\alpha-\beta$ and $\alpha-\beta-\gamma$ filters, respectively, which can be viewed as the archetype tracking filters. The gain of both these filters depends on one parameter only [1], the so-called target manoeuvring index

$$
\lambda=\frac{\sigma_{v} T^{2}}{\sigma_{w}} .
$$

Slightly different $Q$ elements are sometimes used in the models above. Various other models are also used, for example, the well-known third-order Singer model [6], so called CT models for targets with constant turn rate, and other models for different purposes [5]. As for the models above, these models are usually linear and driven by white process noise.

Different adaptive schemes aiming to handle manoeuvring targets have also been developed. It seems that most interest is attended to the so called multiple model approach, where the idea is to use different stochastic models to adapt a filtering algorithm to different manoeuvre situations. The second-order model (4)-(5) with different values of $\sigma_{v}$ is often used for this purpose. Furthermore, (4)-(5) is frequently used for models underlying other complex algorithms. This model is also used in examples to illustrate a certain algorithm. That is, (4)-(5) is a basic model in tracking.

For the issues discussed in the paper, the models (4)-(7) and the corresponding $\alpha-\beta(-\gamma)$ filters are suitable as examples and background. As indicated, they are of basic importance as well. We may have these models and filters in mind for convenience, but the following discussions are relevant for the stochastic approach in general.

\section{Requirements}

In applications tracking filters have to meet certain requirements, in the linear case often formulated by using transfer function concepts. For example, if a tracking filter is to be included in a feedback control system, as is sometimes 
the case, it is natural to require certain frequency response properties to obtain satisfactory closed-loop performance, as usual in control. In open loop, a sufficiently fast response time is usually needed, for example, if the filter estimates are to be used by another system or an operator, as support of some decisions and measures. A suitable dynamic behaviour of a filter is of course required, although this is often understood and not always expressed explicitly. Furthermore, as usual, it is important to make demands on steady-state errors for basic filter inputs like a ramp, rectilinear acceleration, or a circle. Such errors are measures of filter performance for target motion and manoeuvring.

Requirements of this kind are to some extent redundant. Response time and steady-state errors are related to bandwidth, which is defined from the frequency response. Furthermore, the dynamic behaviour can be judged from the frequency response, and there is a conflict between bandwidth and noise sensitivity. Another important parameter is the sampling frequency. Hence, requirements are not always easily formulated, and all the points above are typically not used in a requirement specification. To simplify requirements it is natural to use bandwidth, which gives the following basic requirement: A tracking filter shall have a prescribed bandwidth.

This concentrated and simple formulation can be considered as including several other points as well, since most other filter properties can be brought back in some sense to bandwidth. This basic requirement is important and used in practice. As in other signal processing fields, it is reasonable to consider bandwidth as a primary property also of a tracking filter.

Transfer functions and bandwidth are defined only for linear time-invariant systems, but can still be useful in other cases. As is well-known, linear theory can be applied by linearization around a working point; a local transfer function and bandwidth can then be obtained. This is an indispensable tool in control, and corresponding situations exist for target tracking, for example, when an extended Kalman filter is used.

Even if bandwidth is not used in a nonlinear case, or could be difficult to apply in connection with some complex algorithms, requirements cannot be dispensed with in design, of course. Other performance parameters such as response time and steady-state errors are still relevant.

To further clarify this point, it should be noted that any filter reacts to a change in the input signal with a certain time delay (which is often referred to by saying that the filter has a "time constant"). This is a basic fact for all dynamic systems, of course, and so it is a fact also for tracking filters. In tracking the filter input is the target trajectory positions. A tracking filter cannot react too slowly upon a target manoeuvre input; that is, there will be some requirement in this respect in an application. Such a requirement is usually expressed by using the well-known response time concept, defined from the step response. Even if the definition could be somewhat different, the main point is that response time is a measure of how quickly the filter will react upon an input change.

Requirements in this respect could be quite different in practice. For example, in a track-while-scan application a response time of a couple of seconds might do, while in certain seeker systems for homing guidance the response time should be tenths of a second. That is, response time requirements could differ by a factor ten, and a design procedure must be able to deal with that.

Although clear, it is important to note the simple fact that the requirements, and the performance needed in a specific application, cannot be obtained from a general filter theory. The conditions in the particular application have to be considered, of course, and no general theory can know about that. Requirements often originate from a superior or adjacent system, and it is then up to a filter designer to accomplish appropriate filtering. In fact, an important part of system design, for example in an aerospace project, is about formulating requirement specifications for different subsystems where target tracking might be one.

In practice there are discussions on the interplay between requirements and design, a reasonable change of the requirements may simplify design, and so forth. However, finally there is an agreement on the requirements that the filtering process has to meet. Clearly, a filter designer cannot use some requirements of his own regardless of the application.

It should be noted that to design a filter some requirement is necessary, explicitly expressed or not. Without requirements no design is needed, in fact any filter would do. Design is actually a concept referring to methods and activities used to obtain a system that fulfils the requirements. In related fields such as control theory, signal processing, and electrical circuit design, this is well recognized, and design methods have been developed in accordance with different ways to formulate requirements, where the basic line is transfer function properties. This is in contrast to the situation in target tracking where the significance of requirements is not discussed. In fact, target tracking seems to be the only field where requirements are ignored, when dynamic systems and design are the main topics. Furthermore, in tracking a transfer function foundation is lacking.

\section{The Kalman Filter for Target Tracking}

The Kalman filter characteristics can be considered as twofold: on one hand the filter structure, on the other hand stochastic optimality. The fact that the filter is linear, recursive, and uses a prediction-update state-space configuration may be thought of as structure. It will be argued below that in tracking the structure is the useful part. Stochastic optimality cannot be achieved in practice, and even if it could, it is usually not required in applications.

As a basis for discussion, consider the following formulation of the Kalman filter stochastic optimality.

If the system model (1a) and (1b) is correct and the gain is given by the Riccati equation, then the algorithm (3) gives optimal estimates of the state in the sense that the estimate errors have minimal variance.

This central point of optimality raises questions in the context of target tracking. As for the correctness of the stochastic model, it is clear that the measurement equation (1b) is reasonable; that is, it is usually justified to 
consider measurement errors as additive white noise. Also, the covariance of the noise sequence $w(k)$ may be regarded as known, since the sensor is at the designer's disposal.

The process noise $v(k)$, on the other hand, is questionable. It is of key importance in stochastic approaches, but is usually introduced with little or no justification. Different arguments are occasionally indicated in the literature, which also means different ways to view this noise.

\subsection{Process Noise}

5.1.1. Manoeuvre Process Noise. Although rarely stated, considering process noise as a way to model manoeuvring seems to be the basic attitude. This probably originates from the fact that, in many dynamic models, process disturbances are related to target accelerations and by that to manoeuvring. This is the case in the models (4)-(7) underlying the $\alpha-\beta(-\gamma)$ filters, which is also emphasised by denoting the basic parameter $\lambda$ of these filters as the "target manoeuvring index."

However, interpreting process noise as the source of manoeuvres is not adequate in applications. For example, no aircraft is manoeuvring in such a way that the acceleration can be regarded as white noise, as assumed in the widely used model (4)-(5). It is even difficult to think of the acceleration as a random sequence at all, since a manoeuvre is typically carried out by an acceleration that is constant or slowly varying. Constant radial acceleration is used for circular turns, while slowly varying nearly constant acceleration is relevant for other cases, such as nonperfect circles or evasive manoeuvres. Furthermore, a main problem is that we do not know whether a manoeuvre is going on or not.

What is random about a target acceleration applied for manoeuvring is its magnitude, starting point of time, and duration. White process noise does not correspond to this situation, nor could such noise be regarded as approximations to real situations, which has been argued [1]. Clearly, white noise is far from a reasonable approximation of a constant or a slowly varying quantity. Instead, it is natural to consider the manoeuvring acceleration as an unknown deterministic disturbance.

5.1.2. Aerodynamic Disturbances. An aircraft is exposed not only to applied accelerations for manoeuvring, but also to aerodynamic disturbances such as wind gusts and turbulence. They are supposed to be included in the process noise $v(k)$ and may be considered as white noise without violating the truth too much. However, from tracking point of view, the influence of these disturbances on the target trajectory is normally small, much smaller than typical manoeuvres, and is also reduced by the (auto)pilot. Furthermore, radar is commonly used and its resolution is not good enough to detect the small motions caused by these disturbances. A corresponding covariance for such aerodynamic disturbances will be small, and, consequently, a Kalman filter based on this covariance is likely to be inadequate in practice, as the bandwidth will be too small.
From tracking point of view, it is reasonable to neglect aerodynamic disturbances. In particular, they should not be used as basis for Kalman filters.

5.1.3. Colored Noise. To get what may seem a more realistic model, still within the stochastic formalism, process disturbances are sometimes modeled as the output of a linear system driven by white noise (giving "colored" noise). This is the approach, for example, in the well-known third-order Singer model [6] and in the corresponding second-order Exponential Correlated Velocity (ECV) model. However, by using transfer functions, it has been shown for the ECV case that performance is actually worse by such a model extension [7], contrary to what is generally believed. Thus, what may seem an improvement of the stochastic model does not guarantee an improved Kalman filter. Transfer function analysis of the obtained filter is necessary to see how basic filter properties, such as bandwidth and steady-state errors, are influenced by a stochastic model extension.

5.1.4. Artificial Process Noise. Sometimes a process noise is assumed even if it has no correspondence in the system. This is done to avoid an unreasonable filter. For example, if no process disturbances actually exist, and accordingly no process noise and covariance are assumed, then the Kalman gain will tend to zero. To avoid this, a so called "artificial process noise" with some covariance $Q$ is assumed. At least, this shows that cases exist where a correct process noise model cannot be used to get a reasonable Kalman filter in practice.

It can be argued that white process noise, with a covariance $Q$, is in general to be regarded as "artificial." As discussed above, during straight flight a more suitable covariance is needed than might be suggested by aerodynamic disturbances. For a circle or some other realistic turn, white process noise can be considered as artificial too, as it is not reasonable to consider the disturbance input (causing the manoeuvre) as a stochastic process.

Clearly, this raises further questions about the stochastic model and optimality. However, as will be discussed below, $Q$ cannot be regarded as a model parameter. Instead, it plays the role of a design parameter.

5.1.5. The Markov Argument. A stochastic argument sometimes referred to for introducing white process noise is that it makes the state equations a Markov process. Unless a stochastic state-space system is a Markov process, the state vector is actually not the state in the usual sense that it summarizes the past history of the system (in a probabilistic sense) [1]. This may seem a plausible argument, valid in general for linear stochastic systems.

However, some remarks can be made. First, in many situations, stochastic models are not needed for design. In other fields, for example control, successful design of systems of all kinds has been based on deterministic models. In fact, this is the basic line where no process noise or any appeal to the Markov argument is needed. Clearly, if the purpose is design, there is usually no need to force the Markov 
argument and white process noise into the picture, at least not in many basic cases. This is in contrast to the stochastic approach, where most if not all tracking algorithms are based on stochastic models with white process noise, even in simple basic cases.

If a stochastic approach is nevertheless applied, and if it is accepted that a white process noise has to be introduced, then the question of the correctness of the model arises: is white noise a reasonably correct model of the actual disturbances? As discussed above, and further argued below, in tracking applications this is not the case. Apparently, to make the stochastic state space equations a Markov process, a disturbance model is introduced which is fundamentally incorrect, a strange consequence of the Markov argument. It should also be noted that continuous-time white noise cannot be a correct model of any real signal.

Clearly, the Markov argument is not indispensable and raises further questions about the stochastic approach.

5.1.6. The Unknown Input Argument. To justify the stochastic approach, it has been argued that, if an input is unknown, it should be assumed to be white noise. In [8] it is admitted that "the system (manoeuvring target) does not have a random input," but since the deterministic inputs are unknown "the best course of action is to model the inputs as a random process."

This may be questioned, though. If the process disturbance input is assumed to be white noise with a certain covariance, it determines an estimation algorithm, in the linear case a certain Kalman filter. This makes the noise assumption crucial, and it is far from clear that assuming a certain covariance gives a filter that will be "the best course of action."

5.1.7. A Conclusion. In the discussions above, it has been argued that white process noise is not adequate as a part of the models underlying tracking filters. Aerodynamic forces used for manoeuvring are certainly not white noise. Instead, they should be considered as an unknown deterministic disturbance. Furthermore, different arguments indicated in the literature for white process noise are questionable. In view of this, it may be concluded that there is no process noise in applications.

However, by assuming white process noise in the model (1a) a Kalman filter is obtained, which is probably the main reason for this assumption.

5.2. Optimality. In the discussion above of the stochastic model, it has been pointed out that assuming white process noise is far from reasonable in tracking applications. Of course, optimality may be questioned if the model is severely wrong.

However, it is important to note that even if the model (1a) and (1b) is correct and a mean square optimal filter actually is obtained, such a filter cannot be taken for granted in applications and may even be completely inadequate. Small errors are desirable, but other properties are normally more important. For example, there is a trade-off between error variance and bandwidth. To obtain a required bandwidth (and by that sufficient response time or required steady-state errors for example), a filter with estimation errors above the minimum may have to be accepted. That is, stochastic optimality is questionable also under the perfect conditions of a correct model.

Furthermore, it should be noted that typical requirements in applications would not suggest the use of an "optimal" algorithm based on a stochastic model. For example, to use a filter based on the model (4)-(5), the requirements would include a formulation like: the filter shall be stochastic mean-square optimal, based on the assumption that the target acceleration is white noise. Clearly, such a requirement is not realistic, and so, the use of a stochastic "optimal" algorithm cannot be considered as a response to requirements in this case. The situation is similar for other model cases.

However, despite that most natural objections can be raised, based on well-known facts, the Kalman filter has become the standard basis for target tracking. It is reasonable to ask why: if target motion cannot be modelled appropriately for Kalman filtering, and stochastic optimality is not the purpose, then why use a Kalman filter?

It should be noted that this question is relevant for stochastic algorithms in general, not only for a single Kalman filter. Also more complex algorithms are based on stochastic models with white process noise and aim for mean-square optimality. This makes the question justified in general.

This question is not discussed in the tracking literature. On the contrary, the current tracking paradigm rather prescribes the Kalman filter or some other stochastic algorithm to be used, even in simple basic cases.

5.3. Process Noise as a Design Method. It is an important fact that by introducing a certain process noise a tracking algorithm is determined. For example, if a certain covariance is used for the white process noise in the linear case, then a Kalman filter is obtained. Using another covariance will give some other Kalman filter. The situation is the same for tracking algorithms in general, as they are also based on stochastic models driven by white process noise, and the covariance of this noise determines the algorithm properties.

Introducing process noise can then be viewed as a choice of design method, rather than a model that describes the target behavior. In fact, introducing process noise means that an approach is chosen, where the parameters in the process noise covariance have to be used as design variables in an iterative procedure.

For example, for $\alpha-\beta(-\gamma)$ filter design, different values of the parameter $\sigma_{v}$ have to be tried until a value has been found that fulfills the requirements reasonably well (if possible). The general case is similar: process noise covariance parameters have to be tried in an iterative procedure to design a tracking algorithm. It is noteworthy, though, that such a "trial-and-error" method for design does not seem to be used in the stochastic estimation approach and is not referred to in the tracking literature.

Actually, in the stochastic estimation approach to tracking, design should be understood in the following way: 
introducing white process noise means choice of a particular design method where parameters in the process noise covariance are design parameters.

The design parameters have to be chosen in an iterative "trial-and-error" procedure. This view is also suggested by comparison with optimal control, as discussed in the Appendix.

This conception of design can also be expressed by a simple question: how to choose the covariance for the nonexistent process noise?

In particular, for the models (4)-(7) the question is how to choose $\sigma_{v}$. However, this basic question is not discussed from design point of view in the tracking literature.

Clearly, introducing white process noise for filter design is a somewhat delicate business. It has to be done with care; it cannot, so to speak, be done with an air of innocence.

It should be noted that design of the $\alpha-\beta(-\gamma)$ filter is simplified and more direct, by using the root-locus diagrams and steady-state errors given in $[9,10]$.

5.4. Observers. In contrast to stochastic optimality, the Kalman filter structure is useful. It is natural to use this structure also for some other gain than can be obtained from the Riccati equation, as is the case in the well-known observers. What should be discussed is filter performance for different gains. In any case, in applications a tracking filter has to satisfy the requirements. In this respect an "optimal" gain is no exception and should be regarded as just one case among possible gains.

An observer can be designed by any method that gives a required filter. Since the poles and zeros, and some associated quantities such as bandwidth and response time, are normally checked anyway in a design procedure, it can be argued that pole placement should be used in the first place. Pole placement design of observers is more simple and direct compared to Kalman filters. The Riccati equation is a complicated non-linear matrix equation, the analytical solution of which is known only in certain special cases. Unless such cases are used, analytical expressions for the gain factors are lacking, which makes design and analysis difficult.

Instead, for basic tracking filters an observer approach can be used, including pole placement and transfer function analysis. This has a desirable simplicity, gives valuable insights into performance, and will include commonly used requirements in a natural way.

It should be noted that the steady-state Kalman filter is actually included in the deterministic observer approach, as the Kalman gain can be used in an observer. However, in observers not only the Kalman gain is possible but other gains as well, which could be necessary or useful.

\section{Filter Performance}

6.1. Kalman Filter Performance. In view of the basic importance of the Kalman filter in tracking, a primary question is about its performance: how do we know that a kalman filter, based on an unrealistic stochastic model, is reasonable and satisfies different requirements?
It seems that this basic question is not discussed. The only Kalman filter concept used as performance measure is the error covariance obtained from the Riccati equation. However, as demonstrated in Section 9 for the $\alpha-\beta$ filter, this error covariance can be misleading. In addition, no general relationships seem to be known between the error covariance and other basic filter properties. A bandwidth/covariance relation has been obtained for a special case [7]; otherwise, these questions are not discussed in the literature. It should also be noted that when the Kalman filter covariance is referred to, it is usually assumed that the underlying stochastic model is correct. As this is not the case, there are further doubts about what the filter covariance actually represents. Clearly, the Kalman filter error covariance can be questioned as performance measure.

Besides the fact that a steady-state Kalman filter is stable (under the usual observability and controllability conditions), other basic filter properties-such as bandwidth, response time, steady-state errors, poles and zeros-are not known. That is, important filter properties are not known from the fact that the gain is determined by the Riccati equation. It is still less known, of course, if the requirements for a specific application are satisfied.

Of course, what counts in the end is the performance of the selected filter, not the models or design methods. A linear stochastic model can be seen as a vehicle to obtain a Kalman tracking filter, the properties of which have to be investigated anyway for the application in question. The only input to a tracking filter is the measured target trajectory, which is composed of two parts: the target position and measurement noise. These two inputs can be considered separately, and the output from a noise input can be superimposed on the deterministic output to get the total output (as the filter is linear). Regardless of the design method, basic filter properties can then be obtained, and it is natural to use transfer functions for this purpose. As for other filters, transfer functions can in a simple way give valuable insights also into Kalman tracking filters. This is even more justified if filter requirements are formulated in terms of transfer function concepts, as is often the case in practice.

How to use transfer functions for design and performance evaluation is demonstrated in Section 9 for the $\alpha-\beta$ filter. This approach is also used in [7, 9-13]. It is difficult to see how the results there could be obtained by using "the stochastic estimation approach.”

6.2. Monte-Carlo-RMS Evaluations. When an algorithm is evaluated or when two algorithms are compared, the first order of business should be to check if the requirements are satisfied. However, this is not done for algorithms in the tracking literature, as requirements are ignored there. If, regardless of the requirements, a comparison is to be done anyhow for linear filters, it is natural to check the bandwidth, but in the stochastic approach to tracking this is not done either, as bandwidth is not discussed. The situation is the same for response time. Another most important performance measure is the deterministic steady-state error for a circular turn, a quantity that should have high priority for evaluation and comparison. However, it has not been 
observed until recently [10] that tracking filters can actually give such an error, so it has not been used either.

Instead, in the stochastic estimation approach, the common and as it seems only way to evaluate a suggested algorithm is via Monte-Carlo simulations, using RMS errors as the performance measure. Comparison to another algorithm is often done, and if the RMS measure is smaller for the suggested algorithm, it is said to "outperform" the other.

However, it is clear that this evaluation method is not sufficient. RMS errors may be questioned as performance measure, since requirements are not taken into account, and no relationships seem to be known between RMS errors and other important filter properties, such as bandwidth, response time, and steady-state errors.

Furthermore, as Monte-Carlo-RMS simulation is the only evaluation method in the estimation approach, it is used even in simple cases, such as the $\alpha-\beta$ filter. In no other field would simulations (and certainly not stochastic simulations) be considered necessary or appropriate to obtain performance for a second-order filter with constant coefficients.

In a typical tracking paper an estimation algorithm is proposed, and an example is usually given where RMS errors obtained from Monte-Carlo simulations are presented. In such papers, there are usually no arguments given for the particular choice of the process noise covariance underlying the filter. Second-order models like (4)-(5) are commonly used where a certain $\sigma_{v}$-value is chosen without any discussion. Sometimes the purpose is comparison to other algorithms, which also use some process noise covariance in the models without any justification. The natural question here is: how will the outcome be influenced by other choices of the covariance?

6.2.1. Trajectory Dependence. For linear filters it seems reasonable to ask how bandwidth influences the result of RMS evaluations and comparisons. For simplicity, think of the position filter of the $\alpha-\beta$ filter. This is a low-pass filter, and so it will attenuate input noise. That is, if the input is white noise, then the output variance will be smaller than the input variance, and the attenuation will be stronger if the bandwidth is decreased. For straight flight, the errors are caused only by white measurement noise, and so for this case the errors will be reduced if the bandwidth is decreased, and this will be the case also for the RMS errors obtained by Monte-Carlo simulations. Hence, if the position estimates of two $\alpha$ - $\beta$ filters are compared for straight flight by MonteCarlo-RMS evaluation and one "outperforms" the other in this sense, it is because it has lower bandwidth. This also means that we can always get a "better" filter for straight flight in this sense by just decreasing the bandwidth. But this may be in conflict with the requirements, of course.

However, the $\alpha-\beta$ filter also gives a deterministic steadystate error for a circular turn [10]. For a bandwidth reduction such an error is increased, while stochastic errors caused by white measurement noise are decreased. That is, the size of deterministic and stochastic errors for a turn will respond differently to a bandwidth change. Furthermore, the radius of the turn circle influences the steady-state error; in fact, decreasing the radius will increase the error. Then, for a certain radius, the deterministic steady-state error may dominate the stochastic error, and so some reasonable increase of the bandwidth could be better in RMS sense. On the other hand, for a large radius the situation is more like straight flight, and a filter with smaller bandwidth might be "better." Thus, the choice of trajectory may influence the result of RMS evaluations and comparisons. A further complication is that also the target velocity influences the steady-state error for a turn, as discussed in [10].

Considering these issues, it seems reasonable to ask if the conclusion of RMS evaluations and comparisons of filters depends on the conditions. In particular, we may ask if RMS comparisons of two $\alpha-\beta$ filters can give different results for different trajectories.

If a filter is used that gives no deterministic steadystate circular error, the situation is simplified and more like straight flight. For example, this is the case for a filter based on the Coordinated Turn Model with known turn rate [11]. However, for most filters a steady-state circular error will probably be obtained.

It should also be noted that no one would probably rely on Monte-Carlo-RMS simulations in other fields (e.g., control) where dynamic system properties and design are the topics. And all the more so if it is the only performance evaluation method.

6.2.2. A Conclusion. It cannot be taken for granted that lower Monte-Carlo-RMS errors mean a "better" filter. In any case, it is not reasonable to use RMS errors as the only performance measure. Clearly, RMS-errors are insufficient as performance measure.

Monte-Carlo-RMS simulations are normally used without any discussion or justification. The literature is full of such evaluations and comparisons of algorithms. They should be viewed with some suspicion.

Remark 1. A tracking algorithm is usually considered and referred to as being "optimal." Then there should be no need for comparisons to other algorithms, of course. Furthermore, other proposed algorithms are also claimed to be optimal, a strange situation. However, such comparisons merely show again that "optimal" cannot be interpreted in the usual sense as "best possible" here. As noted above, "optimal" is just a notion for a particular design method.

\section{Multiple Model Approach}

The basic idea in the multiple model approach is to use different stochastic models, each assumed to represent a particular manoeuvring situation, and use an adaptive estimation algorithm based on these models. The purpose is to handle manoeuvring targets. Several algorithms have been proposed on how to actually accomplish such adaption. To discuss the issues the following archetype case is suitable.

7.1. A Basic Case. Consider a scenario where the target moves in a horizontal plane with constant velocity on 
a straight line course followed by a circular turn, a standard trajectory in tracking. Assume (in each Cartesian coordinate) two linear stochastic models, one for the straight flight and the other for the turn. For simplicity, take the second-order model (4)-(5) with two different process noise levels $\sigma_{v}$. Consider the $\alpha$ - $\beta$ filters for these two models and assume that switching between these filters is based on filter errors using some criterion, according to which a manoeuvre has started and is going on. This scenario and filtering can be considered as the multiple model approach in its most basic setting. It is suitable for discussion purposes, but is relevant also for more complex situations and algorithms.

Some questions arise for this case. First and most basic: why use adaptive filtering at all? The reason seems to be that a stochastic "optimal" solution is desired, based on the assumption that the different models actually are correct descriptions of the manoeuvring situations. Then a switch is needed when the target makes a manoeuvre, in order to use the "optimal" filter for the current mode (straight flight or turn).

However, as usual, referring to optimality is questionable. The stochastic models are not correct, a least-square optimal criterion is not relevant, and requirements are not involved, as discussed earlier. Then, also in this case, searching for the "optimal" solution is not adequate in the first place.

Furthermore, an adaptive algorithm is not needed in many cases when requirements enter in the design procedure. For example, suppose that an adaptive algorithm meets the requirements during the turn (as should be the case); that is, suppose that the turn filter actually satisfies the requirements during the turn. Then the turn filter can be used for the whole trajectory, provided that the requirements are the same for straight flight. No adaption is really needed; only the turn filter can be used, and this filter satisfies the requirements.

This is a natural idea when a multiple model algorithm is suggested, also since the filter intended for the turn normally has higher bandwidth than the straight flight filter (as higher covariance is used in the turn model). The higher bandwidth during straight flight (using the turn filter) is actually desirable, as the response to a manoeuvre will be faster. We can compare with a servo system: if there is no input to a servo, it would not be advisable to reduce the bandwidth, as the servo must be able to respond quickly (in accordance with some requirements) to a possible input. And, of course, it is favourable to use a single filter if possible, as it has a desirable simplicity compared to an adaptive algorithm.

Clearly, in many situations in applications, different requirements are not needed for straight flight and turning. But if this is the case, some adaptive filtering would be necessary. However, it should be noted that even with different requirements a single filter might do. Consider the case that a single $\alpha-\beta$ filter is used. This filter gives a deterministic steady-state error for a circular turn, in contrast to zero error during straight flight [10], while the errors caused by measurement noise are the same in both situations. So, using a single $\alpha$ - $\beta$ filter means smaller errors during straight flight. Thus, it may happen that different requirements for straight flight and turning could be fulfilled with only one filter.

A crucial part in adaptive filtering is the manoeuvre detection, which in some way is based on filter errors. Independent of how this is accomplished, several measurements are needed to establish that a manoeuvre has actually started and is going on. That is, it takes some time before the turn filter is activated and the higher bandwidth is obtained. Adaptive filtering can then be inadequate during the beginning of a manoeuvre, as the response might be too slow.

7.2. The IMM Estimator. In the basic case above, fixed filters are considered and the switching between them is assumed to be the result of a decision based on filter errors. Several alternative approaches have been suggested for the switching and filtering mechanism, in order to obtain a more flexible and "continuous" adaption where the filters in some way run in parallel. The most popular seems to be the so called IMM (interacting multiple model) estimator where a "soft switching" and filtering is accomplished by a certain probabilistic "mixing" [1]. This is a somewhat complex estimation algorithm where, among other things, so called transition probabilities are also needed. These probabilities are further design parameters that have to be selected. It should be noted that the second-order model (4)-(5) is commonly used in this approach.

However, also the IMM estimator raises questions. In fact, the issues discussed for the basic case above are still relevant. As always, the search for stochastic optimality is questionable. Also "smooth" switching takes time, which means that a filtering process intended for the turn cannot be activated at once. As usual, requirements are ignored, so the need for IMM filtering is not clear in the first place. In fact, also for the IMM approach it is obvious that, multiple model filtering is not needed if the requirements are the same for straight flight and turning.

Furthermore, only the insufficient Monte-Carlo-RMS criterion is used as performance measure when an IMM algorithm is evaluated.

An IMM algorithm example is discussed in [1, Section 11] and in [14]. The conditions are similar to the basic case above: a trajectory consisting of straight flights and circular turns, where the target motion is modelled by two secondorder models (in each coordinate) given by (4)-(5) with different process noise levels. An IMM algorithm for this situation is compared to a single Kalman filter, based on the same model, using a process noise level between those in the IMM models. Comparison is evaluated in terms of RMS errors obtained by Monte-Carlo simulations. It is argued in [14] (and in [1]) that the IMM estimator performs better than the single Kalman filter if the tracking index $\lambda>0.5$.

However, the criterion $\lambda>0.5$ contradicts the recommendation $\lambda<0.4$, based on a well-known rule of thumb for the bandwidth/sampling frequency ratio in discrete time system design [10]. Of course, a rule of thumb should not be taken too literally, a larger $\lambda$ may work, but some care should then be taken about the dynamic behaviour of the filter. Furthermore, the contradiction is based on only one investigation [14] of a particular case, and the 
bandwidth expression used is an approximation. However, it is noteworthy that, regardless of other objections, the IMM estimator does not give improved performance in MonteCarlo-RMS terms for the case in [14] if the design rule $\lambda<$ 0.4 is accepted.

\section{Some Further Viewpoints}

8.1. Stochastic Errors. Measurement of the target position is input to a tracking filter. Stochastic filter errors are then caused by the measurement noise, as this is the only noise input to the filter. The magnitude of these filter errors is determined by two factors: the filter and the measurement noise level. A reasonable tracking filter (not necessarily a Kalman filter) means low-pass filtering, and so it attenuates white measurement noise; for example, the position estimates are at least more accurate than the measurements. Furthermore, the accuracy is improved in this respect if the filter bandwidth is reduced. However, if the filter is required to have a certain bandwidth, stochastic errors caused by white measurement noise cannot be reduced below some bound by the filter. In this sense, stochastic errors are not a question of filtering in the first place; instead, the measurement noise is the main factor.

The measurement noise level is determined by several contributions, such as sensor resolution, inertial stabilisation (of airspace sensors), and image processing (all together causing "jitter" in electrooptical systems). Sensor resolution is the fundamental parameter here, since other parameters, for example, some measure of inertial stabilisation performance, should if possible be matched to the resolution; otherwise, the resolution cannot be fully utilized. Hence, the conclusion is as follows: for the size of stochastic errors, sensor resolution is the basic property.

Although this observation is obtained by having linear filters in mind, it should be relevant for other cases as well. Normally, resolution is determined by state of the art of sensor technology and cannot be further improved.

8.2. Sensor Exchange. Sometimes the sensor is exchanged in a system. For example, a new sensor could be introduced to upgrade range performance or because of change of technology (e.g., from IR to radar). The sensor resolution, and by that the measurement noise level $\sigma_{w}$, could then be either increased or decreased. Suppose that a suitable Kalman filter is already in use, based on some white process noise model, when the sensor is exchanged. For simplicity, think of the $\alpha-\beta(-\gamma)$ filter for a certain value of $\sigma_{v}$. If the process noise is considered, not as contributing design parameters, but as an adequate or at least approximate model for actual target manoeuvres, then we should keep this model for filter design, of course, even if the sensor is exchanged. For the $\alpha-\beta(-\gamma)$ filter, this means that we should use the same $\sigma_{v}$. If also the sampling interval $T$ is the same, then the only parameter to be changed because of a sensor exchange is $\sigma_{w}$.

But another Kalman filter will then be obtained. In the $\alpha-\beta(-\gamma)$ case, increased $\sigma_{w}$ means decreased manoeuvring index $\lambda$ (8), which in turn gives decreased filter bandwidth
[9]. Then requirements concerning bandwidth, response time, or steady-state errors are perhaps no longer satisfied. Decreased $\sigma_{w}$ means increased bandwidth, which might be favourable, but could also be unsuitable; for example, in practice the bandwidth should not be too high compared to the sampling frequency $1 / T$.

From the manoeuvring index $\lambda$, it is seen that to obtain the same $\alpha-\beta(-\gamma)$ filter and by that the same performance for deterministic target manoeuvres, $\sigma_{v}$ has to be changed by the same amount as $\sigma_{w}$. Thus, if $\sigma_{v}$ is regarded as a manoeuvre model parameter, then to obtain the same filter performance for manoeuvring, we have to assume that the target manoeuvring has changed if the sensor is exchanged. Clearly it is a strange situation, since a target certainly makes its manoeuvres irrespective of the sensor properties. This further emphasises that $\sigma_{v}$ has to be regarded as a design parameter, not a parameter to model target manoeuvring.

Remark 2. This discussion has some relevance for the case that the sensor parameter $\sigma_{w}$ is time varying. When such a variation is known and included in the model and the Kalman algorithm is used, then the filter gain will be changed accordingly. In the stochastic estimation approach, this is considered to be a great benefit, as the algorithm is still "optimal" in mean-square sense even in the presence of such a parameter variation.

However, it is not clear that changes of the filter gain caused by $\sigma_{w}$ variations are appropriate. When the gain is adjusted, filter properties are changed. We may think of these changes in terms of bandwidth, response time, or steadystate errors, at least if the $\sigma_{w}$ time variation is reasonable. Then some requirements are perhaps no longer satisfied. Filter properties for target manoeuvres are usually more important than a change in the measurement accuracy.

Again we can compare with servo systems. It is not advisable to change the bandwidth of a servo if the variance of an additive white noise input has changed.

This points out-once more-that the concept of "optimality" is questionable.

8.3. The Benedict-Bordner Paper. In basic books on target tracking, transfer functions and related concepts are mentioned only in passing, if at all. These matters are treated in a few papers, though. A noteworthy reference is BenedictBordner [15]. In this early paper, design of the general $\alpha-\beta$ filter (not the Kalman filter version) is discussed. By an optimisation procedure, a simple relation between $\alpha$ and $\beta$ is obtained $\left(\beta=\alpha^{2} /(2-\alpha)\right)$ by which a reasonable dynamic behaviour is guaranteed. As the authors note: for all $\alpha$ the filter will be slightly underdamped. Then BenedictBordner make the following important observation: "This analysis does not specify the optimum value of the single remaining parameter $\alpha$; this would be impossible. The choice of $\beta=\alpha^{2} /(2-\alpha)$ is essentially one of damping factor, which can be optimised. The remaining choice of $\alpha$ is one of bandwidth. Selection of an a value must depend upon the system application."

As discussed above, this is a fact of basic importance in tracking filter design. It is of course relevant also for a Kalman 
filter and is of corresponding importance for other "optimal" algorithms as well.

8.4. Estimation versus Design. It should be noted that state estimation is not equivalent to filter design. Actually, there is a difference between estimation and design, and much of the discussions and conclusions above can be viewed in the light of this fact.

Suppose that a system is described by the stochastic model (1a) and (1b). Optimal estimates in mean-square sense of the state variables are then given by the Kalman algorithm (3). If the purpose is to obtain the state variables, but not otherwise use the algorithm, then we have an estimation situation. For the sake of illustration, imagine that (1a) and (1b) is a physical system where one of the state variables is a physical quantity, the knowledge of which is of general interest in physics. Measurements $y(t)$ can then be collected whenever it would be convenient, and an estimation algorithm can be processed on any computer whenever we like. The algorithm can be iterated to obtain estimates as close to steady-state optimality as desired, and normally it is not important how long time it takes; the main purpose is to obtain god estimates. Furthermore, there is no need to consider the estimation algorithm as a "filter" that responds to input signals in a certain specified way.

However, if the algorithm is to be used "on line" as a filter, in order to process input signals in a dynamic environment in an application, then requirements enter the picture, as always in applications. This is the case also in tracking. For example, the filter response time might be a parameter that has to be taken into account and most likely other requirements as well, as discussed above (Section 4). This means a design problem. Then no filter designer, participating in an application project, can seriously say for example "do not mind the requirements" or "a steady-state error does not matter" or "an aircraft makes a turn by using white noise accelerations" (it would be considered as a joke).

The main points of the paper can now also be summarized in the following way: the task of producing a tracking filter is not an estimation problem. It is a design problem.

The difference between estimation and design is not recognized or discussed in tracking. Surprisingly, the approach in tracking is that the best way to design a filter is to use an estimation algorithm (usually based on a fundamentally incorrect stochastic model). Such an algorithm is even claimed to be "the optimal filter", and this is asserted without any consideration to requirements. As discussed in the paper, this is far from reasonable.

\section{The $\alpha-\beta$ Filter}

9.1. Background. The $\alpha-\beta$ filter is the steady-state Kalman filter for the two-state model (4)-(5). It is the basic tracking filter, discussed below by using transfer functions. Some of the results can be found in [9] and [10], also based on transfer functions. An exposition of these issues here is justified for various reasons.

It is a suitable and easily grasped example of the transfer function approach. Together with [7, 9-13], it illustrates the benefits of this approach. Its relation to stochastic estimation is also demonstrated.

The $\alpha$ - $\beta$ filter is discussed in many references using the stochastic estimation approach. However, no design of the $\alpha-\beta$ filter has in fact been done, as the necessary choice of the process noise parameter $\sigma_{v}$ (to meet requirements) is not discussed. By using transfer function parameters and performance measures given below (such as bandwidth and steady-state errors), design to meet commonly used requirements can be done. Independent of the design issues, such performance parameters are also of basic interest of course.

The steady-state $\alpha-\beta$ Kalman filter can be determined directly from the algebraic Riccati equation. It is also obtained as the steady-state limit of the time-dependent Kalman algorithm applied to (4)-(5). It should be noted that, if the transient part of the algorithm is included and even if the transient is considered to be important in a particular application, the properties of the steady-state filter are still of interest. After all, the time-dependent filter tends to the steady-state filter, and it is not likely in an application that the transient will be satisfactory if the steady-state filter is not. In any case, also when the transient part is included, the process noise parameter $\sigma_{v}$ has to be chosen to give a suitable filter, that is, the filter has to be designed. For this purpose the steady-state filter properties should give valuable guidance.

The situation is similar in this respect if parameter variations or nonlinearities are present in the model (4)-(5). Steady-state filters for different parameter values, or for models obtained by linearization, could then contribute valuable information. Furthermore, (4)-(5) is also used as models underlying more complex algorithms, and so the steady-state filter should be of interest in such cases too.

Finally, it should be noted that $\alpha-\beta$ filters are actually used in basic applications.

9.2. Transfer Functions. The gain of the $\alpha-\beta$ filter is denoted as

$$
L=\left[\begin{array}{l}
\alpha \\
\frac{\beta}{T}
\end{array}\right] .
$$

Then the transfer functions of the $\alpha-\beta$ filter are [9]

$$
\begin{gathered}
\frac{\hat{x}_{1}(k \mid k)}{y(k)}=\frac{(\alpha z-\alpha+\beta) z}{z^{2}+(\alpha+\beta-2) z+1-\alpha} \stackrel{\text { def }}{=} H(z), \\
\frac{\hat{x}_{1}(k \mid k-1)}{y(k)}=\frac{(\alpha+\beta) z-\alpha}{z^{2}+(\alpha+\beta-2) z+1-\alpha}, \\
\frac{\hat{x}_{2}(k \mid k)}{y(k)}=\frac{(\beta / T)(z-1) z}{z^{2}+(\alpha+\beta-2) z+1-\alpha}, \\
\frac{\hat{x}_{2}(k \mid k-1)}{y(k)}=\frac{(\beta / T)(z-1)}{z^{2}+(\alpha+\beta-2) z+1-\alpha} .
\end{gathered}
$$


Equations (10) and (11) are the update and prediction position filters respectively. Equations (12) and (13) are the corresponding velocity filters; they can be interpreted as lowpass filtering of the derivative of $y$, since $(z-1) / T$ represents differentiation. $\alpha$ and $\beta$ depend only on the manoeuvring index $\lambda$ (defined in (8)) and can be expressed in closed form [1]:

$$
\begin{gathered}
\alpha=-\frac{1}{8}\left(\lambda^{2}+8 \lambda-(\lambda+4) \sqrt{\lambda^{2}+8 \lambda}\right), \\
\beta=\frac{1}{4}\left(\lambda^{2}+4 \lambda-\lambda \sqrt{\lambda^{2}+8 \lambda}\right) .
\end{gathered}
$$

For small $\lambda$ useful approximations are $\alpha \approx \sqrt{2 \lambda}$ and $\beta \approx \lambda$.

The filter is linear, and the input signal $y$ is the sum of two parts: target positions and measurement noise. These two inputs can be treated separately, and their outputs give the total filter output by superposition. All basic filter properties can then be obtained from the transfer functions.

9.3. Poles and Zeros. The poles and zeros are given in [9] as root-locus diagrams with respect to $\lambda$. From these diagrams, it is seen that the positions of the two poles correspond to a damping factor $\zeta \approx 0.7$. The bandwidth is determined by the pole-zero locations and their distance to $z=1$, in combination with the sampling interval, that is, the filter bandwidth is determined by $\lambda$ and $T$. In applications $\sigma_{w}$ and $T$ are normally given quantities, fixed by the sensor, in which case the bandwidth is determined by $\sigma_{v}$. It is seen that the bandwidth increases with $\sigma_{v}$. The bandwidth $\omega_{B}$ and $\lambda$ are approximately related as

$$
\omega_{B} T \approx 2 \sqrt{\lambda}
$$

This relation is given in [10] where also further discussion of bandwidth can be found.

A suitable range of $\lambda$-values for design can be judged from the root-locus diagrams in [9], since it is good design practice to place the poles not too far from $z=1$. For example, a choice in the interval $\lambda<0.5$ is reasonable. If considerably higher values are needed, it is an indication that the bandwidth requirement might be too high compared to the sampling frequency. The dynamic behaviour could then be unsuitable. It is seen that the zeros have reasonable locations relative to the poles for realistic $\lambda$-values. Hence, these filters should have suitable dynamic behaviour; for example, a step response will have reasonable overshoot without oscillations.

These valuable insights into the properties of the $\alpha-\beta$ filter cannot be obtained from the underlying stochastic model, the Kalman filter error covariance, or RMS errors obtained from Monte-Carlo simulations. Transfer functions, with their poles and zeros, are needed for these matters. As usual, transfer functions are simple and transparent and reveal important properties that are hidden by the stochastic state space formalism. The significance of pole-zero positions for different filter properties is further discussed in [16].
9.4. Steady-State Errors. Steady-state errors for different standard inputs are performance measures for basic manoeuvres. They can be obtained from the transfer functions as usual. From (10) and (11) it is seen that a step as well as a ramp input will be reproduced with no steady-state position error, both for the update and prediction filters. For an acceleration input $y(t)=a t^{2} / 2$, the position errors are

$$
\begin{array}{lr}
\frac{1-\alpha}{\beta} a T^{2} & \text { (update filter) } \\
\frac{1}{\beta} a T^{2} & \text { (prediction filter). }
\end{array}
$$

Here $(1-\alpha) / \beta$ and $1 / \beta$ are monotonic decreasing functions of $\lambda$. Thus, for a given $T$, the acceleration errors decrease with increasing $\lambda$.

From the transfer functions (12) and (13), it is seen that for a step input the velocity estimates will be zero in steady state. That is, if $y(k)$ is a step, the velocity estimates tend to zero after an initial transient, as should be the case. Furthermore, for a ramp input $y(t)=V t$, both the update and prediction velocity estimates will give the correct velocity value $V$ in steady state. An acceleration input $y(t)=a t^{2} / 2$ yields the velocity errors

$$
\begin{array}{ll}
\frac{2 \alpha-\beta}{2 \beta} a T & \text { (update filter) } \\
\frac{2 \alpha+\beta}{2 \beta} a T & \text { (prediction filter). }
\end{array}
$$

These errors are monotonic decreasing functions of $\lambda$. That is, for a given $T$, the velocity errors decrease with increasing $\lambda$.

Steady-state errors for a circular turn can be obtained from the frequency functions, as discussed in [10]. The position error is mainly in the radial direction, given for small $\lambda$ by

$$
e_{r} \approx \frac{a_{r}}{\sigma_{v} / \sigma_{w}},
$$

where $a_{r}$ is the (radial) acceleration. In [10] a better approximation is also given if $\lambda$ is not small, as well as exact expressions for the errors. However, the basic parameters involved are clearly seen in (19). The error is determined by the target acceleration divided by the ratio of the stochastic model parameters, a ratio that is essentially the square of the filter bandwidth. As usual, the error is reduced if the bandwidth is increased. It is seen in (19) how to choose the ratio of the stochastic parameters in order to keep the error below a certain bound.

The error (19) is of interest in many applications. It is actually determined by the process noise parameter $\sigma_{v}$ when $\sigma_{w}$ is a given sensor parameter. If this circular error is relevant in a specific application, but a requirement in this respect is ignored, then a certain choice of $\sigma_{v}$ could give unacceptable errors. Without a requirement, taken into account in the design, there is no guarantee that this will not happen.

This is the case also if the underlying model (4)-(5) is "true," of course, that is, even if the parameters $\sigma_{w}$ and $\sigma_{v}$ 


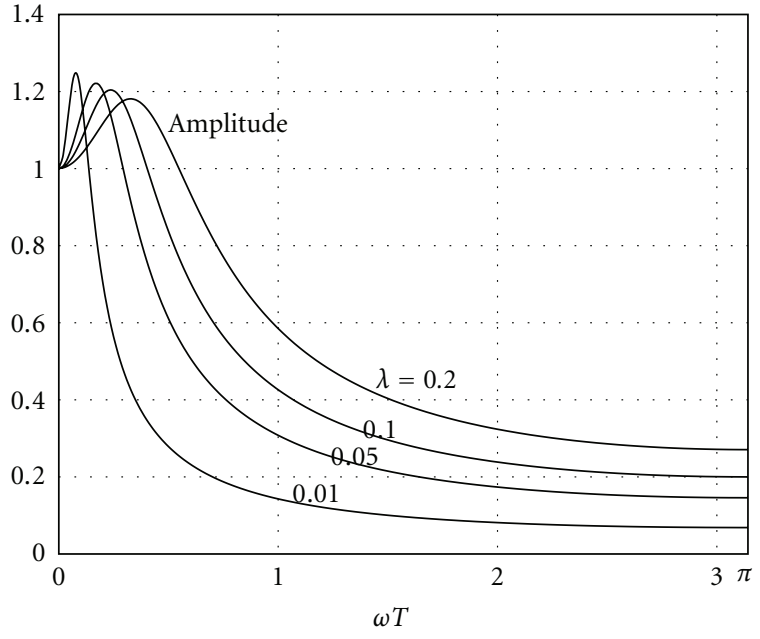

FIGURE 1: Amplitude frequency functions.

are "true." Although this can never happen, it is the perfect ideal situation in the estimation approach, in which case the Kalman algorithm is considered to be "optimal," that is, the "best possible" filter. However, it may happen that the "true" parameter values give an unacceptable error (19). That is, even under perfect conditions in terms of estimation, "the optimal filter" could be useless in a certain application. Then the only way to obtain acceptable errors (i.e., to fulfil a requirement) is to use another $\sigma_{v}$-value.

Remark 3. Filter requirements can usually be fulfilled for different design parameter values, that is, for different choices of the process noise covariance. For example, an upper bound on the error (19) can be fulfilled for different $\sigma_{v}$-values. This freedom in the parameter $\sigma_{v}$ can be used to reduce the stochastic errors (caused by measurement noise). The choice of $\sigma_{v}$ can then also be viewed as a stochastic error minimization problem, subject to the requirement constraints.

However, there is not much room for optimization here. $\sigma_{v}$ determines the filter bandwidth, and it is good design practice that the bandwidth should not be higher than necessary. One reason for this is to keep the noise influence down. Then, most likely, there is not much to be gained by choice of the only design parameter $\sigma_{v}$ in an optimal procedure.

The situation is similar if more design parameters are available in the process noise covariance. Also in such cases, a transfer function with required bandwidth is the goal. As the stochastic errors are determined by the transfer function, it seems that not much will be gained by using more design parameters either.

So, in practice, it is fair to say that if a filter has been designed that satisfies requirements on dynamic properties then the stochastic errors are also essentially determined.

9.5. Frequency Response. To give a frequency response example, consider the update position transfer function $H(z)(10)$.

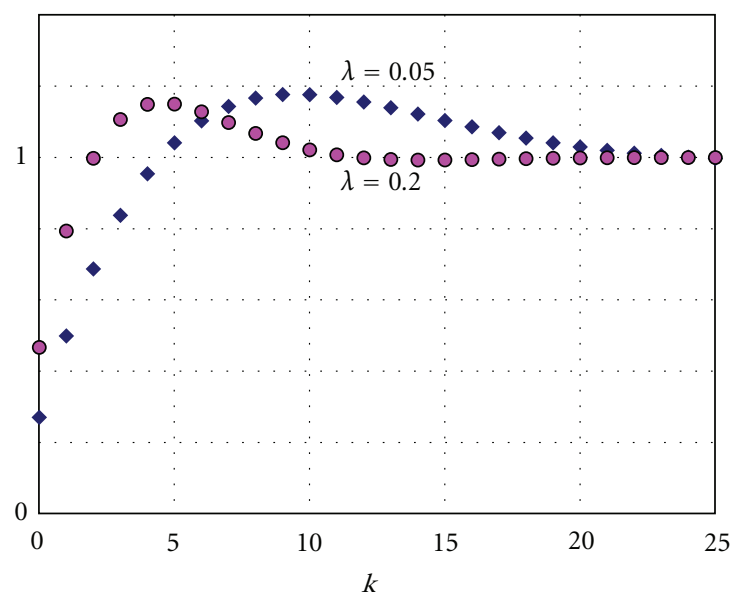

FIGURE 2: Step responses.

The amplitude function, the absolute value of $H\left(e^{i \omega T}\right)$, is shown in Figure 1 as function of $\omega T$ (up to the Nyqvist frequency $\pi / T$ ) for different $\lambda$-values.

It is seen that the resonance peak value is about 1.2 , which is suitable and means that the step response has a reasonable overshoot without oscillations. The same conclusion was obtained from pole-zero considerations, as should be the case. The peak value dependence on $\lambda$ is small. Defining bandwidth in the usual way, as the frequency $\omega_{B}$ at which the amplitude has decreased by the factor $1 / \sqrt{2}$, the bandwidth can be determined for the cases in the diagram. For a given $T$, the bandwidth increases with $\lambda$.

In applications the sampling frequency should be well above the bandwidth, to obtain good or expected dynamical behaviour. As a rule of thumb, a factor 10 may be used, that is, the relation $\omega_{B} \leq 2 \pi / 10 T$ is desirable. Such a rule is useful to judge if a required bandwidth is reasonable in relation to the sampling frequency or for the choice of sampling frequency. Of course, a rule of thumb can be discussed; other factors are also used in the rule. These issues are further discussed in [16].

9.6. Step Response. Step responses for $H(z)$ (10) are shown in Figure 2 for two $\lambda$-values. If the same sampling interval $T$ is used in the two cases, it is seen that the response is faster for the higher $\lambda$-value as expected, since for a given $T$ the bandwidth increases with $\lambda$. Also as expected, the dynamic behaviour is otherwise similar for the two cases. The overshoot is about $20 \%$ and no oscillations occur, in accordance with the resonance peak values. For clarity, only two $\lambda$-cases are shown, but the result is similar for other cases: faster response if $\lambda$ is increased, otherwise similar for different $\lambda$-values. Clearly, we may have a response time requirement such that the filter for $\lambda=0.2$ will do, but not the filter for $\lambda=0.05$, although both are "optimal."

9.7. Measurement Noise Input. Consider the case where measurement noise is the only input to the filter that is, let $y(k)$ be a stationary zero-mean white sequence with standard 
deviation $\sigma_{w}$. The output variance can then be conveniently obtained from the transfer functions by the iterative method in [16]. For the general second-order case a closed form expression is available, which can be directly applied to the transfer functions (10)-(13), yielding

$$
\begin{gathered}
\frac{\sigma_{1 u}^{2}}{\sigma_{w}^{2}}=\frac{2 \alpha^{2}+2 \beta-3 \alpha \beta}{\alpha(4-2 \alpha-\beta)} \stackrel{\text { def }}{=} \Delta_{1}, \\
\frac{\sigma_{1 p}^{2}}{\sigma_{w}^{2}}=\frac{2 \alpha^{2}+2 \beta+\alpha \beta}{\alpha(4-2 \alpha-\beta)}, \\
T^{2} \frac{\sigma_{2 u}^{2}}{\sigma_{w}^{2}}=T^{2} \frac{\sigma_{2 p}^{2}}{\sigma_{w}^{2}}=\frac{2 \beta^{2}}{\alpha(4-2 \alpha-\beta)} \stackrel{\text { def }}{=} \Delta_{2} .
\end{gathered}
$$

$\sigma_{1 u}$ and $\sigma_{1 p}$ are the standard deviations of the update and prediction position estimates, respectively. Equation (20) gives the output/input variance ratio of the transfer function (10), while (21) gives the corresponding ratio of the transfer function (11). For the velocity transfer functions (12) and (13), the same ratios (22) are obtained where $\sigma_{2 u}$ and $\sigma_{2 p}$ are the standard deviations of the update and prediction velocity estimates, respectively. The ratios (20) and (22) are shown in Figures 3 and 4 as functions of $\lambda$. It is seen that $\Delta_{1}<1$, that is, the position update is always more accurate than the measurements.

9.8. Kalman Algorithm Covariance. Denote the update stead$y$-state error covariance matrix of the Kalman algorithm by $\left[p_{i j}\right]$. It can be shown [1] that

$$
\begin{gathered}
p_{11}=\alpha \sigma_{w}^{2}, \\
p_{22}=\frac{\beta}{T^{2}} \frac{\alpha-\beta / 2}{1-\alpha} \sigma_{w}^{2} .
\end{gathered}
$$

Thus, $p_{11}$ is the variance of the error of the update position estimate, as obtained from the Kalman algorithm, and $p_{22}$ is the corresponding variance of the error of the velocity estimate. These quantities are often referred to as measures of the state estimate accuracy.

In Figures 3 and 4, these algorithm variances are compared to the variances (20) and (22) obtained from measurement noise. It is seen that $p_{11}$ and $p_{22}$ always exceed $\sigma_{1 u}^{2}$ and $\sigma_{2 u}^{2}$, respectively. That is, the Kalman algorithm variances overrate the variances of the errors caused by measurement noise. The differences could be considerable. For example, if $\lambda=0.1$ (a reasonable value in practice), then $p_{22}=$ $3.6 \sigma_{2 u}^{2}$. That is, for the velocity error, the standard deviation obtained from the Kalman algorithm could be nearly twice the standard deviation caused by measurement noise.

In applications the actual stochastic errors are caused only by measurement noise, as this is the only noise input to the filter. Thus, $p_{11}$ and $p_{22}$ could be misleading as measures of stochastic errors. If they are utilised in a system for some reason (e.g., for gate control in an imaging sensor), they should be used with care.

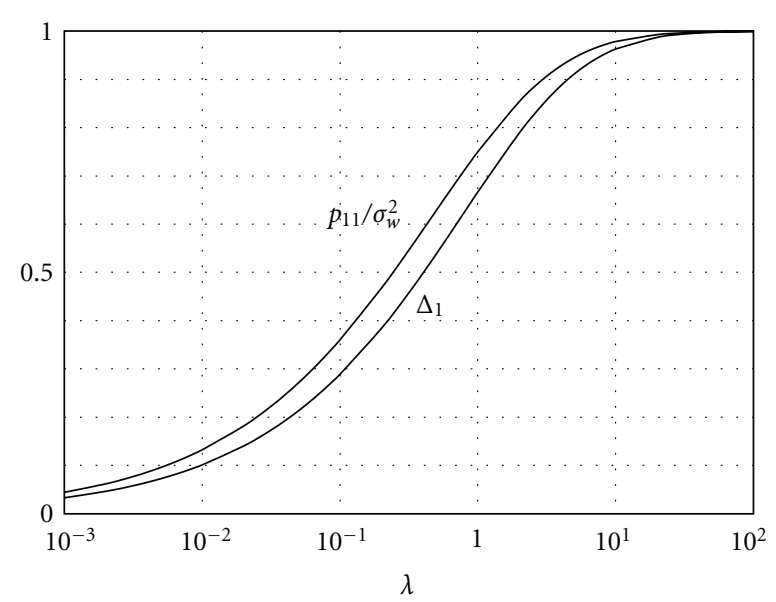

Figure 3: Noise ratios $\Delta_{1}$ and $p_{11} / \sigma_{w}^{2}$ as functions of $\lambda$.

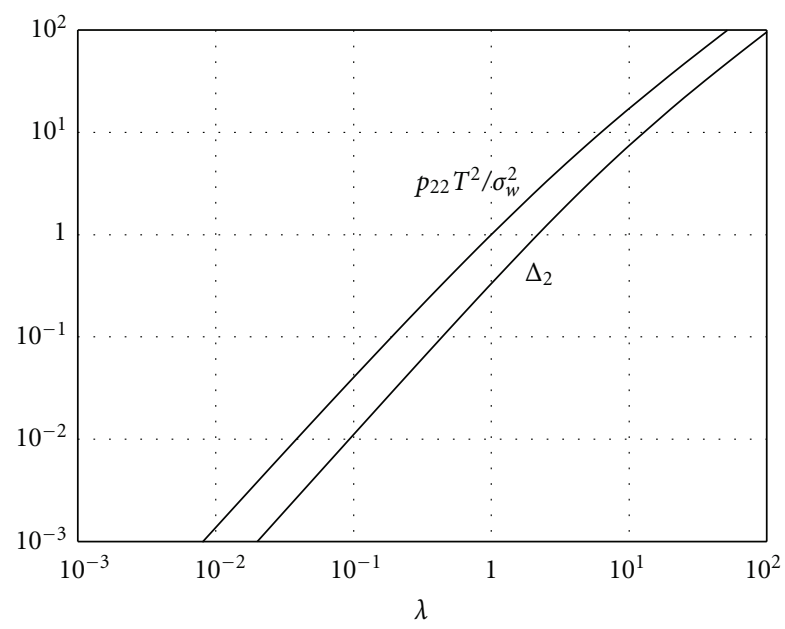

Figure 4: Noise ratios $\Delta_{2}$ and $p_{22} T^{2} / \sigma_{w}^{2}$ as functions of $\lambda$.

\section{Conclusions}

The foundations of tracking filter design have been discussed. It is argued that the basis of the present stochastic estimation approach is questionable in applications. For simplicity and clarity, the issues are discussed with focus on the Kalman filter, but the arguments and conclusions are relevant in general. The $\alpha-\beta$ filter is used as illustrative example.

10.1. Basic Issues. To summarize the basic issues of the stochastic approach in a simple way, consider the following items:

(i) the stochastic models,

(ii) the estimation algorithms,

(iii) requirements,

(iv) RMS performance measure.

These items are discussed in the paper. Briefly, the following can be said. 
The stochastic models are fundamentally incorrect (no aircraft makes a turn by using white noise accelerations). Surprisingly, an estimation algorithm based on such a model is used and even claimed to be "the optimal filter." Even more surprising, this is done without taking requirements into account. Furthermore, the only performance measure is RMS errors, obtained by Monte-Carlo simulations, a stochastic measure that is clearly insufficient.

These issues are not discussed in the tracking literature. Requirements are ignored in the stochastic approach, and the other points are accepted without any discussion or justification.

Furthermore, a basic question in the stochastic approach is the following: how to choose the covariance for the assumed process noise?

In particular, in the commonly used model (4)-(5) the question is how to choose $\sigma_{v}$. This is the crucial design question in the stochastic approach, but it is not discussed as such either.

It is also a remarkable fact that, in contrast to all other signal processing fields, in tracking a transfer function oriented foundation is lacking.

10.2. Main Points. Besides the basic items above, some main points in the paper can be listed as follows.

(i) Optimality, in the usual stochastic Kalman filter sense, can be questioned in tracking applications for two basic reasons: white process noise is not adequate as a model for manoeuvring, and stochastic meansquare optimality is neither relevant nor required in applications.

(ii) Requirements are necessary for design, but this is ignored in the stochastic approach to tracking. Without requirements any filter would do. Requirements concerning bandwidth, response time, and steadystate errors are common in practice.

(iii) Design is a notion referring to methods and activities used to meet the requirements.

(iv) Design parameters: the elements of the covariance of the process noise are design parameters, not model parameters. They have to be chosen to meet the requirements.

(v) Basic filter properties such as bandwidth, response time, and steady-state errors cannot be obtained from the Kalman filter covariance or from Monte-CarloRMS simulations. Transfer functions are needed for these important performance parameters.

(vi) There is no process noise: aerodynamic forces applied for manoeuvring should be considered as deterministic, and different arguments for white noise in the literature are questionable.

(vii) RMS errors are insufficient as performance measure.

(viii) Multiple model filtering is not needed if the requirements are the same for straight flight and turns. Furthermore, if the bandwidth/sampling frequency ratio is taken into account, cases exist where the IMM estimator does not give improved performance in RMS terms.

(ix) The error covariance in the Kalman filtering algorithm can be misleading, even as a measure of stochastic errors. It is not known in general how this covariance is related to important performance parameters such as bandwidth.

(x) Sensor resolution is the main parameter for the level of stochastic filter errors.

(xi) Structure should be regarded as the main point of the Kalman filter, not optimality. Using the structure, an observer approach can be used. This is a convenient, less complicated design method, where requirements can be taken into account in a natural way.

(xii) Benedict-Bordner pointed out, a long time ago, that all gain parameters in a tracking filter cannot be determined by a general optimal procedure, in view of the fact that a certain bandwidth is required in applications.

(xiii) There is a difference between estimation and design.

\section{Appendix}

\section{A. Comparison with Optimal Control}

As is well known, the optimal state estimation problem, leading to the Kalman filter, is mathematically equivalent to the optimal control problem, in which case a quadratic performance index is minimised by linear state feedback. In both cases the solution is given by the same type of Riccati equation, and the involved matrices just have different meanings $[1,16]$. It is then to be expected that these two fields have much in common, also from a practical design point of view.

The purpose in optimal control is the minimisation of a performance index, which represents a cost. However, this optimisation is usually considered and used as a design method among others, where the performance index is not thought of as a cost or cannot be interpreted as one. Optimal control design is an iterative process, carried out by choosing different matrices in the performance index, each of which gives a feedback solution. To see if a solution is suitable, the closed-loop system is preliminary evaluated, typically by checking the step response. This procedure is continued until a satisfactory system is obtained. The matrices in the performance index are design parameters used to "tune" a suitable feedback system [17], and this procedure is in fact a "trial-and-error" method for design.

The parallel to optimal state estimation is obvious. The matrices in the control performance index correspond to the covariance $Q$ and $R$ in the model for state estimation. This correspondence can be seen by a direct comparison of the Riccati equations for the two cases. Thus, pursuing the comparison, $Q$ and $R$ can be considered as design parameters, which are chosen to obtain a filter that satisfies the requirements. If $R$ is considered as a known model 
parameter, which is reasonable for a given sensor, then the elements of $Q$ are the design parameters. For the models in Section 3, this leaves us with $\sigma_{v}$ as the only design parameter if the sampling interval $T$ is fixed.

Sometimes in the optimal control case, the performance index actually represents a cost that shall be minimised. A "true" optimal control system will then be obtained. For state estimation, this corresponds to the case where the stochastic model is correct, and the objective actually is to minimise the mean-square estimation error. However, as discussed above, this situation is not valid for state estimation in tracking, and so there is no parallel to optimal control in this case. Considering $Q$ and $R$ as design parameters is the only relevant interpretation. That is, comparison with optimal control leads to the following conclusion: For a Kalman Tracking Filter, Q and R Are Design Parameters.

If $R$ is considered as a known model parameter, then $Q$ is the design parameter.

A consequence of this conclusion is that referring to a Kalman filter as "optimal" is not adequate in tracking. Although the filter originates from an optimal estimation criterion, the necessary use of the stochastic noise parameters for design makes it comparable to other design methods. In fact, as in control, "optimal" is only a notion for a particular design method where the gain is obtained from a Riccati equation by choice of the matrix $Q$.

It should be noted that optimal control design was not developed or needed for design of SISO (single input, single output) systems in the first place, in which case classical control methods can be used. Instead, an important reason for the success of optimal control was the application to multivariable systems. When introduced, it was the only available design method in practice for this purpose. Transferred to state estimation, this means that there is no real need to use an optimal-based procedure for design of basic tracking filters.

Furthermore, optimal control of SISO systems is well known in terms of classical control concepts for the closed loop, such as transfer functions, stability margins, and pole-zero positions. Relations between the performance index and pole positions have also been clarified [17]. The corresponding issues have not received any attention in the optimal filtering case.

\section{References}

[1] Y. Bar-Shalom, X. Rong Li, and T. Kirubarajan, Estimation with Applications to Tracking and Navigation, Wiley, New York, NY, USA, 2001.

[2] S. S. Blackman and R. Popoli, Design and Analysis of Modern Tracking Systems, Artech House, Norwood, Mass, USA, 1999.

[3] Y. Bar-Shalom and X. Rong Li, Estimation and Tracking: Principles, Technics, and Software, Artech House, 1993.

[4] Y. Bar-Shalom and T. E. Fortmann, Tracking and Data Association, Academic Press, New York, NY, USA, 1988.

[5] X. R. Li and V. P. Jilkov, "Survey of maneuvering target tracking. Part I: dynamic models," IEEE Transactions on Aerospace and Electronic Systems, vol. 39, no. 4, pp. 1333-1364, 2003.

[6] R. A. Singer, "Estimating optimal tracking filter performance for manned manoeuvring targets," IEEE Transactions on
Aerospace and Electronic Systems, vol. 6, no. 4, pp. 473-483, 1970.

[7] B. Ekstrand, "Design aspects of a continuous-time tracking filter," IEE Proceedings: Control Theory and Applications, vol. 151, no. 1, pp. 1-5, 2004.

[8] I. W. Osborne, Y. Bar-Shalom, and T. Kirubarajan, "Radar measurement noise variance estimation with several targets of opportunity," IEEE Transactions on Aerospace and Electronic Systems, vol. 44, no. 3, pp. 985-995, 2008.

[9] B. Ekstrand, "Poles and zeros of $\alpha-\beta$ and $\alpha-\beta-\gamma$ tracking filters," IEE Proceedings: Control Theory and Applications, vol. 148, no. 5, pp. 370-376, 2001.

[10] B. Ekstrand, "Steady-state circular errors for basic tracking filters," IEE Proceedings: Control Theory and Applications, vol. 153, no. 4, pp. 413-418, 2006.

[11] B. Ekstrand, "Continuous-time tracking filters for the coordinated turn model," Bentham Open, The Open Aerospace Engineering Journal, vol. 2, pp. 1-9, 2009.

[12] B. Ekstrand, "Tracking filters and models for seeker applications," IEEE Transactions on Aerospace and Electronic Systems, vol. 37, no. 3, pp. 965-977, 2001.

[13] B. Ekstrand, "Analytical steady-state solution for a continuous time Kalman filter," IEEE Transactions on Aerospace and Electronic Systems, vol. 21, no. 6, pp. 746-750, 1985.

[14] T. Kirubarajan and Y. Bar-Shalom, "Kalman filter versus IMM estimator: when do we need the latter?" IEEE Transactions on Aerospace and Electronic Systems, vol. 39, no. 4, pp. 1452-1456, 2003.

[15] T. R. Benedict and G. W. Bordner, "Synthesis of an optimal set of radar track-while-scan smoothing equations," IRE Transactions on Automatic Control, vol. 7, no. 4, pp. 27-32, 1962.

[16] K. J. Åström and B. Wittenmark, Computer Controlled Systems, Prentice-Hall, 1997.

[17] B. D. O. Anderson and J. B. Moore, Optimal Control. Linear Quadratic Methods, Prentice-Hall, 1971. 

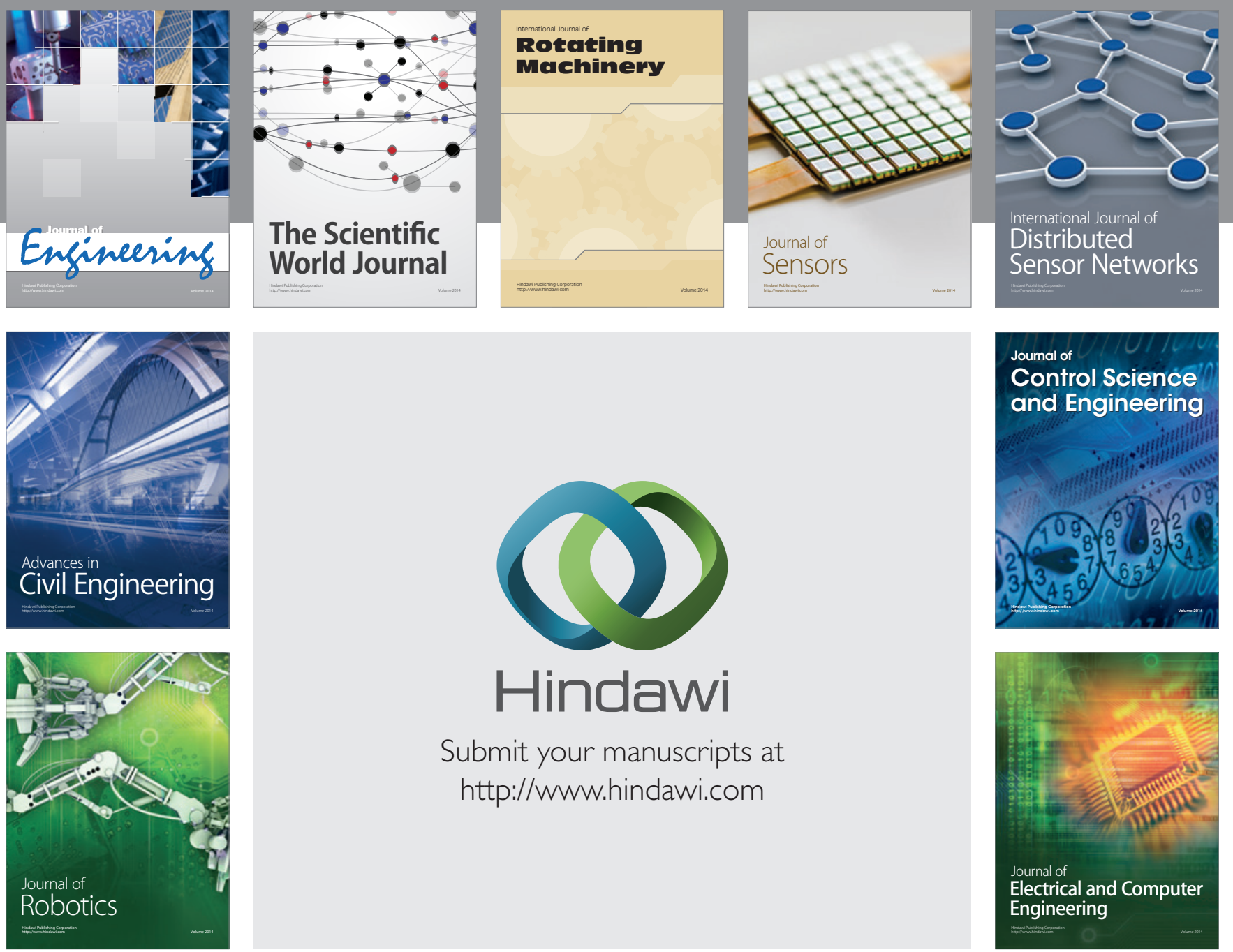

Submit your manuscripts at

http://www.hindawi.com
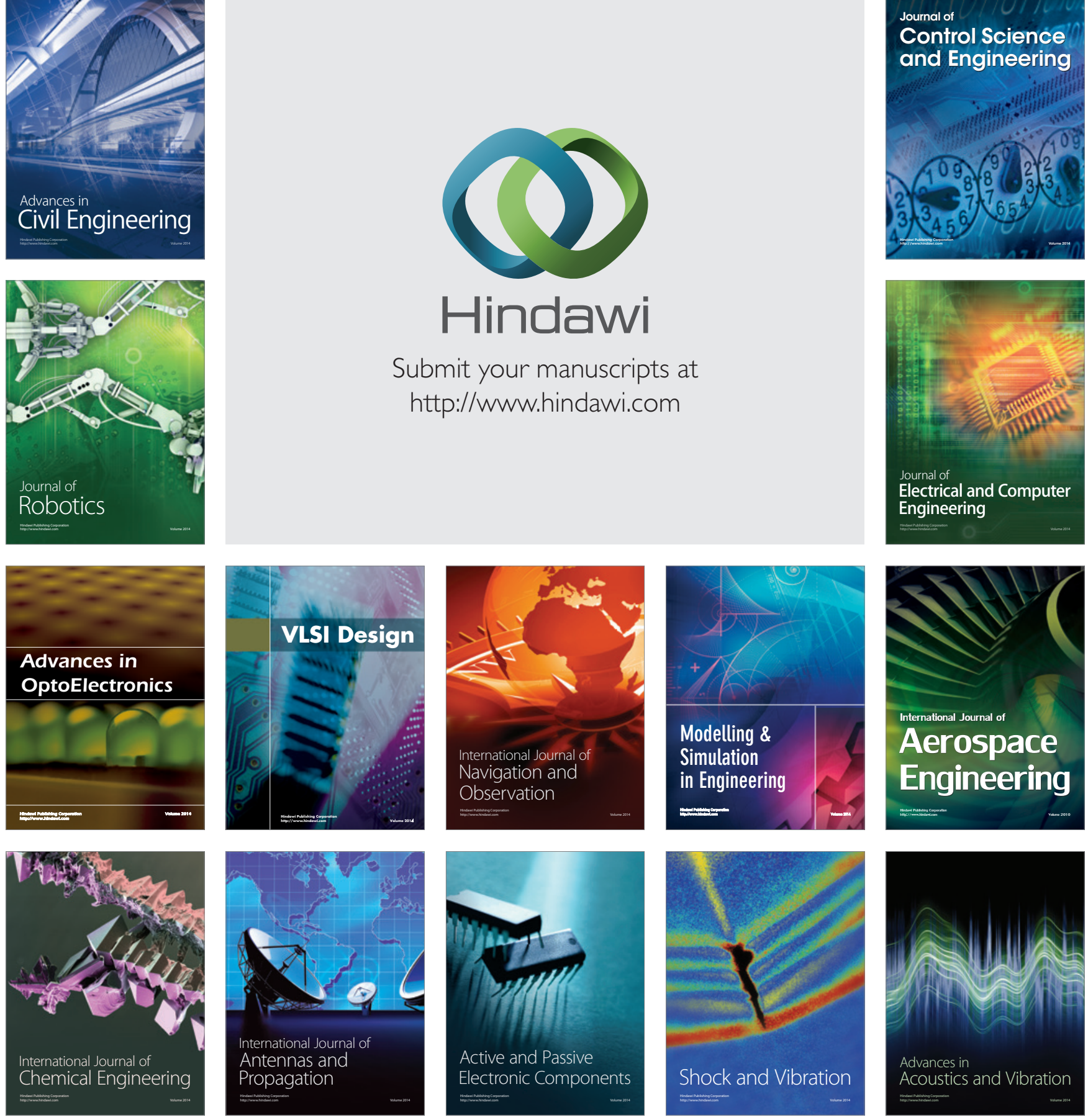